\title{
| FUNCIÓN PROFESIONAL DEL PEDAGOGO EN CENTROS HOSPITALARIOS COMO ÁMBITOS EDUCATIVOS EXCEPCIONALES
}

\author{
(PROFESSIONAL ROLE OF PEDAGOGUE IN HOSPITAL CENTERS \\ AS EXCEPTIONAL EDUCATIONAL FIELDS)
}

María José Latorre Medina

y Francisco Javier Blanco Encomienda

Universidad de Granada

\section{RESUMEN}

En la literatura científica especializada en el campo de la preparación profesional de los pedagogos se vislumbran espacios y escenarios de inserción profesional bien diferentes a los tradicionales. El presente trabajo pretende ser una contribución en esta dirección, analizando el papel del pedagogo en las aulas hospitalarias. A lo largo del artículo, se justifica cómo la presencia del pedagogo en estos dominios no convencionales se erige como una necesidad vital y se esbozan cuáles son las funciones, tareas y competencias propias o de uso profesional de los pedagogos hospitalarios. Por último, se evidencia uno de los problemas del campo profesional que requiere respuesta desde la formación inicial, abogándose por una cualificación más acorde con la labor del pedagogo en estos ámbitos educativos excepcionales.

\section{ABSTRACT}

In specializing scientific literature in the field of professional preparation of pedagogues, some spaces and scenarios of professional insertion are emerging, which are very different from traditional ones. This paper seeks to make a contribution in this direction, analyzing the role of pedagogue in hospital classrooms. Throughout the article, the presence of pedagogue in these unconventional domains as a vital necessity is justified and it is shown which the functions, tasks and own or professional skills of hospital pedagogues are. Finally, one of the problems of the professional area that requires response 
from initial training is evidenced, advocating by a qualification that should be more consistent with the work of pedagogue in these exceptional educational fields.

\section{FUNDAMENTOS Y BASES DE PARTIDA. UNA VISIÓN DESDE LA TEORÍA}

Resulta incuestionable que, en los últimos años, la pedagogía ha ampliado de manera increíble su espectro de actuaciones, habiendo surgido además muy diversas especializaciones dentro de la misma carrera de cara al mundo laboral como son pedagogía social, laboral, escolar, de personas mayores, diferencial, hospitalaria, especial, tecnología educativa y un largo etcétera que expanden más aún aquello que muchos autores han definido como Pedagogía.

Centrándonos en una de estas especialidades, concretamente, en la Pedagogía Hospitalaria, González y Polaino (1990) afirman que fue a principios de la década de los 70 cuando los pedagogos hicieron su aparición en el ambiente hospitalario de los denominados países desarrollados (Estados Unidos, Canadá y Centro-Norte Europa) con diversos fines y bajo el paraguas de múltiples teorías. La presencia de estos profesionales y la proliferación de diversas actuaciones pedagógicas en el tratamiento de enfermos hospitalizados ha ido creciendo paulatinamente sin que, en muchos casos, dichas prácticas hayan tenido un reflejo real en los fundamentos teóricos de la pedagogía, ni mucho menos en la producción científica asociada a la misma. Han sido y son actuaciones que forman parte de aquéllas que están encaminadas a atender las necesidades más propias de la persona, que bien por no ser objeto directo de la actuación médica o por no ser parte de las competencias propias del personal sanitario, no son cubiertas por el servicio hospitalario. Y es la educación la que se hace garante de ellas, llegando a conformar poco a poco un cuerpo disciplinar conocido como "Pedagogía Hospitalaria".

\subsection{Aproximación al concepto de Pedagogía Hospitalaria}

Haciendo especial incidencia en los ámbitos de una educación no formal, fuera de los esquemas convencionales de trabajo de los pedagogos, Ortiz (1999) nos perfila una nueva vía ya abierta de la pedagogía, la hospitalaria, desde una clara perspectiva multiprofesional y de compromiso familiar y social. En su trabajo, la autora va estableciendo relaciones entre diferentes constructos que nos facilitan su comprensión. Las etiquetas conceptuales que asocia son las siguientes: 


\section{Educación no formal / educación en el hospital}

En la década de los noventa, Ortiz (1999) revela que autores como González-Simancas y Trilla, al plantear las áreas de actuación de la educación no formal, incluyen contenidos, ámbitos y colectivos específicos como son la educación sanitaria y la pedagogía hospitalaria. De este modo, dejan patente la relación existente entre la sanidad y la educación no formal. No obstante, es la pedagogía hospitalaria la que, desde su óptica, contempla las áreas educativas que, cada vez más, van asumiendo los centros hospitalarios, en relación a: compensar el retraso académico por la falta de escolaridad; dar contenidos educativos en los tiempos libres fuera del tratamiento médico y atender a las secuelas derivadas del propio estado de enfermedad.

\section{Necesidades educativas especiales / necesidades asistenciales especiales}

Por un lado, desde el ámbito educativo, Ortiz (1999) recuerda que el nuevo concepto de necesidades educativas especiales, acuñado por el Comité Warnock en Inglaterra en 1978, hacía referencia a la situación especial en que un alumno puede encontrarse en cualquier momento de su vida, pudiendo precisar de actuaciones también especiales. Es un concepto relativo, transitorio e interactivo en el sentido de que dependen tanto de las condiciones personales como de las características del medio (en contra del concepto de déficit del modelo médico). Asimismo, llama la atención sobre que, por aquél entonces, la amplitud del término se hacía ya extensible al niño enfermo, al mencionarse en el Informe Warnock que "hay niños que se ven obligados a permanecer en un hospital durante períodos más o menos largos de tiempo", planteándose la situación del niño hospitalizado, la necesidad de que existiera un aula hospitalaria y, lo más importante, la necesidad de formar a un profesorado motivado, sensible y especializado.

Sin duda, es la situación que vive el niño hospitalizado de ruptura con su ritmo de vida, de separación de sus amigos, de su familia..., en general, su estado carencial (en lo afectivo, social, físico), lo que promueve la implantación de actuaciones educativas especiales que reajusten el equilibrio perdido en el niño por un factor indeseable, la enfermedad. A tal efecto, la formación de los profesionales tiene mucho que ver con la que se plantea en la educación inclusiva, bajo el lema de una escuela abierta a la diversidad, que abarca a colectivos más concretos o ámbitos más específicos.

Y, por otra parte, desde el ámbito médico, en la literatura al uso también se pone de relieve la nueva definición de niños con necesidades asistenciales especiales en los centros hospitalarios. En general, son aquellos niños que sufren un proceso crónico de tipo físico, del desarrollo, conductual 
o emocional, o que están expuestos al riesgo de padecerlo y que, además, requieren servicios sanitarios y complementarios en un sentido amplio, en el que se incluye toda una serie de servicios para mantener o mejorar su salud y capacidad funcional, como son: servicios terapéuticos (fisioterapia, logopedia...), servicios de apoyo familiar; equipo y material, etc.

Aunando uno y otro campo, el médico y el educativo, en torno al niño enfermo, se dan la mano las necesidades educativas especiales y las necesidades asistenciales especiales; y la compensación de ambas no tiene otra forma de ser que la de una acción interprofesional sobre el niño, la familia, los profesionales del hospital y los del centro escolar. La colaboración médicopedagógica y familiar (López y Fernández, 2006) se erige como el punto de confluencia que abre el camino hacia una pedagogía propia del hospital.

\section{Aula inclusiva / aula hospitalaria}

Gándara y Junquera (2006) parten de la base de que la escuela forma parte de la actividad habitual del niño, resultando indispensable para su desarrollo armónico. Si esto es así para todos los pequeños, ante una situación de enfermedad grave y larga, la escuela pasa a convertirse en uno de los pilares fundamentales para prevenir el aislamiento y pasividad a que tiende todo alumno con una hospitalización prolongada, continua o intermitente.

En otro de sus trabajos, Ortiz (1998) recoge que los niños hospitalizados, al igual que el resto de escolares, tienen el mismo derecho a recibir una adecuada atención educativa en las aulas de apoyo que existen en los hospitales llamadas "aulas hospitalarias" que, por responder a las necesidades específicas que presentan los niños enfermos, son proclamadas como "aulas inclusivas” (León, 2001). Estos espacios educativos excepcionales se establecen como áreas abiertas y flexibles, donde el paciente pediátrico puede acudir libremente, ausentándose siempre que lo requiera su asistencia sanitaria (López y Fernández, 2006).

Normalmente ubicadas en la planta de pediatría, González (2004) dibuja estos espacios como habitaciones amplias, alegres, bien ventiladas y luminosas, sumamente atractivas, llenas de colores, libros, juegos, material docente... Desde el punto de vista de los familiares y profesionales, las aulas hospitalarias son espacios físicos absolutamente necesarios en los hospitales para el bienestar de los niños enfermos. Por ello, deben representar un lugar seguro y normalizador para estos alumnos, donde, además de la función educativa, vean o tengan para ellos un significado relacional, ocupacional e integrador. Tal como se desprende del estudio realizado por Barrueco, Calvo, De Manueles, Martín y Ortiz (1997), las aulas hospitalarias son clave para 
dar contenido educativo a los ratos de ocio, para recuperar el retraso académico y, sobre todo, como vía de enriquecimiento personal y social.

López y Fernández (2006), por su parte, definen las aulas hospitalarias como centros de enseñanza creados por el Ministerio de Educación y Cultura, puestas a disposición de los niños enfermos, en diferentes hospitales de España. Con ellas, se pretende atender el derecho que todo niño tiene a recibir una educación general, incluso cuando sea necesaria su hospitalización, al mismo tiempo que se le proporciona un espacio idóneo dentro del hospital, en el que puede relacionarse con otros niños con los que compartir sus propias experiencias, ayudando así a superar con mayor rapidez los problemas de salud causantes de su ingreso en un centro hospitalario.

En definitiva, la Pedagogía Hospitalaria se configura como aquél cuerpo disciplinar que se dirige expresamente a la atención y optimización de la educación de los niños enfermos que están en un hospital, sea cual sea la edad y el estado físico o mental, intentando mejorar sus condiciones de vida. Su finalidad principal es capacitarles para poder vivir mejor, contribuir al modo en que afrontan y aceptan una enfermedad, sus consecuencias inmediatas, la perspectiva de cambios que conlleva en su vida y los tratamientos que de ella se derivan (González y Polaino, 1990). Según Olivares, Méndez y Ros (2002), es la pedagogía la que, en el contexto hospitalario, puede ayudar a paliar los efectos psicológicos, sociales y educativos que toda hospitalización puede comportar, tanto sobre el paciente pediátrico como sobre su familia.

\subsection{La pedagogía hospitalaria en el marco de una educación inclusiva}

De entrada, en el trabajo informado por Grau (2001) se recogen los numerosos puntos en común que tienen la pedagogía hospitalaria y la educación especial, en cuanto que ambas suponen una acción multidisciplinar y consideran que las dificultades de aprendizaje y los problemas de salud no pueden ser abordados exclusivamente desde la pedagogía o la medicina. Por un lado, la educación especial se ocupa de los alumnos con necesidades educativas especiales, es decir, de aquéllos que requieren de la dotación de medios especiales de acceso al currículo, un currículo especial o modificado, y una atención a la estructura social y al clima emocional y afectivo en que se desenvuelve la acción educativa. Por otro, la medicina se centra en los niños con necesidades asistenciales especiales, esto es, de aquéllos que sufren una enfermedad crónica y requieren servicios sanitarios o complementarios (fisioterapia, logopedia, terapia ocupacional, intervención precoz, cuidados a domicilio, servicios sociales...). Y en la pedagogía hospitalaria confluyen 
estas dos necesidades, las educativas y las asistenciales; de ahí la necesaria acción interdisciplinar (Castillo, 2005; López y Fernández, 2006).

Una simple mirada por la documentación científica publicada al respecto nos permite constatar, efectivamente, que la pedagogía hospitalaria debe asumir los principios defendidos por la educación inclusiva, etapa final del proceso seguido por la educación especial, que es vista como avance, mejora y superación de la integración escolar. Los trabajos realizados por Caballero (2007) y Grau y Ortiz (2001) avalan lo que decimos. De atender a las necesidades educativas especiales del niño se ha pasado a respetar la diferencia, la excepcionalidad, como una característica de su manera de ser persona, y a soslayar todos los prejuicios sociales, culturales, religiosos y profesionales que impiden acceder al conocimiento de la realidad única, original e insustituible de cada ser humano (Ortiz, 2000). La escuela inclusiva parece ser la opción más eficaz para dar apoyos naturales en cualquiera de los ambientes en que se encuentre el niño enfermo, puesto que arbitra todas las medidas necesarias para poder dar la bienvenida al alumno hospitalizado y atenderle también en el sistema educativo ordinario y en el domicilio familiar durante la convalecencia.

\section{LA ACCIÓN PEDAGÓGICA EN AMBIENTES HOSPITALARIOS. UNA APROXIMACIÓN A LA PRÁCTICA}

Con el propósito de justificar la necesidad y posibilidad de la acción pedagógica en estos contextos educativos excepcionales, hemos seguido los trabajos de Castañeda (2006) y Castillo (2006). Para Castañeda (2006), es la concepción de la educación como base de los procesos de mejora de las personas y de la atención educativa como contribuyente fundamental de la salud integral de las mismas lo que justifica la pertinencia de que exista el servicio pedagógico dentro de la atención educativa hospitalaria. Según Castillo (2006), las distintas administraciones han intentado dar una respuesta educativa a las necesidades especiales y específicas que presentan los niños hospitalizados en dos sentidos: por un lado, tratar de compensar los problemas que se derivan de una hospitalización más o menos prolongada y, por otro, facilitar los recursos necesarios para realizar la compensación. No podemos olvidar que estos niños, además de pacientes de un hospital, siguen siendo alumnos con el mismo derecho a la educación que los demás.

En este marco, la figura del pedagogo hospitalario se convierte en un elemento decisivo para el ajuste psicológico, social y educativo del niño enfermo, actuando también de intermediario entre el personal médico-sanitario, la familia y su colegio. Su perfil, se va elaborando con la práctica diaria 
de esta actividad y el apoyo de todos (Fernández, 2000). Pero a pesar de este papel crucial que el pedagogo puede desempeñar dentro del equipo multiprofesional en el que se canaliza su rol, convirtiéndose en centro neurálgico de la actuación educativa que se desarrolla en los hospitales, es el profesional menos valorado dentro de dicho escenario laboral, no sucediendo así con la figura del maestro, del educador social, del psicólogo y menos aún, del profesional/es de la medicina implicados en la recuperación del niño enfermo. Además, las funciones educativas en las aulas de los centros hospitalarios están asignadas en la actualidad solamente a maestros, cuando éstos precisan de un apoyo permanente del que depende la calidad de la actividad educativa desarrollada.

Esta situación ha despertado la voz de alarma de multitud de autores e investigadores, entre ellos, Borja, Fortuny y Fuentes (2003) y Castañeda (2006), reivindicando el papel que debe tener la pedagogía en el contexto hospitalario, del cual ha sido privada en la mayoría de los casos. La última autora aboga por un reconocimiento explícito de las funciones, tareas y competencias que son propias de los pedagogos y que les permiten ejercer su labor educativa en este dominio profesional, como miembro integrante del "equipo pedagógico multidisciplinar" responsable de la atención hospitalaria al niño enfermo. Para ello, Borja, Fortuny y Fuentes (2003) puntualizan que debe ser, durante el período de formación universitaria, cuando se planteen, dentro del campo de actuación de los pedagogos, formas de intervención educativa tanto en el ámbito de la educación formal como no formal.

\subsection{Labor del pedagogo hospitalario}

\subsubsection{Sujetos a los que atiende}

Los principales destinatarios de la Pedagogía Hospitalaria son alumnos, niños y adolescentes, del sistema educativo que por razón de enfermedad permanecen hospitalizados. Cómo hacer que estos alumnos no sólo no pierdan, en lo posible, su ritmo escolar sino que, en la propia enfermedad, se vean asistidos eficazmente por la acción educativa se convierte en labor principal del pedagogo hospitalario.

"El pedagogo hospitalario —afirma Ortiz $(1999,110)$ — se ve abocado a educar en la enfermedad". Y educar en la enfermedad exige conocerla como una situación especial, con todas sus implicaciones, puntos débiles y fuertes, posibilidades y carencias y realidad subyacente. Por tanto, si ésta es una función básica del pedagogo en este escenario profesional, cabe plantearse en qué consiste y cómo ha de hacerlo. 
En primer lugar, parece ser primordial conocer cuál es la situación que vive un niño enfermo en el hospital. En la documentación existente se recoge que el niño vive una situación de ruptura con su ritmo de vida, de separación de sus amigos y de su familia, de aislamiento, de experiencias desconocidas en un medio hostil, en principio. Sin entrar en la enfermedad concreta que padezca, el niño vive en un estado carencial en lo afectivo, social y físico (Palomo, 1995).

Castillo (2006) sostiene que el hecho de que estos alumnos sean pacientes de un hospital les impide la regular asistencia a sus colegios y les provoca, además de las molestias propias de la enfermedad, una situación de miedo y estrés. Afortunadamente, los niños ingresados son alumnos de sus centros y, en la mayoría de los casos, su paso por el aula del hospital va a ser una circunstancia temporal. En relación con la duración de la hospitalización, se distinguen entre los alumnos los siguientes grupos: a) alumnado de larga hospitalización: son aquellos niños que permanecen ingresados más de 30 días a lo largo del curso escolar; b) alumnado de media hospitalización: niños cuya duración en el hospital se sitúa entre los 15 y los 30 días anuales; y c) alumnado de corta hospitalización: aquéllos que permanecen menos de 15 días en un año.

Barrueco, Calvo, De Manueles, Martín y Ortiz (1997), fruto de una investigación realizada en el distrito universitario de Salamanca, nos informan, desde la perspectiva de los propios padres y profesionales, de las situaciones consideradas de riesgo y susceptibles de apoyo en el contexto del niño enfermo. Entre ellas: la salida de un lugar seguro como es el medio familiar hacia un lugar desconocido como es el hospital; la separación de la familia en quien confía para pasar a manos de desconocidos sin saber por qué, hasta cuándo y para qué; la ruptura con la vida cotidiana, la escuela, el barrio para estar aislado en una habitación del hospital; el paso de una actividad frenética marcada por sus iguales a sentirse en la cama, a solas consigo mismo, con todo el tiempo disponible para pensar; el haber perdido un estado físico saludable para sentirse mal, acobardarse o deprimirse y no saber cómo va a evolucionar, etc.

Asumiendo que dichas situaciones pueden perjudicar al desarrollo integral del menor hospitalizado, el pedagogo hospitalario debería tener en cuenta tanto los efectos negativos derivados de la enfermedad, de cara a paliarlos, como las consecuencias positivas derivadas de la misma para ser potenciadas y mantenidas (Ortiz, 1999). En cuanto a los aspectos negativos de la enfermedad, que son precisos eliminar o compensar, ha de saber que: a) en el momento del ingreso, el niño puede reaccionar con irritabilidad, gritando, llorando, rebelándose; luego se vuelve hosco, retraído, triste y finalmente se resigna y se adapta; y b) durante la estancia en el hospital, el niño sufre una serie de trastornos como consecuencia de la separación del am- 
biente familiar, de su estancia en un medio extraño y de la carencia de relaciones afectivas adecuadas como son: los sentimientos de culpa y ansiedad, las fantasías y temor a la mutilación, el abandono, la muerte, etc. Y respecto a los aspectos positivos o beneficios secundarios derivados de la enfermedad, susceptibles de optimizar y potenciar, ha de asumir que la enfermedad: a) es una oportunidad para que el niño exprese sus sentimientos y pida que sus necesidades sean satisfechas; b) promueve una comunicación más abierta en el seno de la familia y crea una atmósfera más saludable; y c) con ella se aprende a decir "no" y a adquirir nuevos y mejores hábitos en áreas como la nutrición, el sueño, ejercicio, etc.

\subsubsection{Objetivos a trabajar en el campo profesional}

"Ante todo son niños, con todo su potencial de desarrollo, al que hay que dar el apoyo educativo para que su desarrollo evolutivo se vea mínimamente afectado por su enfermedad" (Gándara y Junquera, 2006, 16). Si la meta principal del pedagogo hospitalario es proporcionar una atención educativa de calidad a los niños enfermos hospitalizados, de cara a prevenir y evitar la marginación de su proceso educativo, son objetivos básicos a trabajar los siguientes (Castillo, 2006):

- Proporcionar apoyo afectivo al niño y paliar su déficit emocional.

- Tratar de reducir el déficit escolar.

- Disminuir la ansiedad y demás efectos negativos desencadenados como consecuencia de la hospitalización.

- Mejorar su adaptación y ajuste a la hospitalización y a su situación de enfermedad.

- Mejorar la calidad de vida del niño hospitalizado.

- Fomentar la actividad, procurando que el niño ocupe provechosamente su tiempo.

- Cultivar la natural alegría infantil y las relaciones sociales.

- Atender a la formación del carácter y la voluntad del niño, en el sentido de incitar su esfuerzo y animarle al trabajo.

Listado de objetivos que López y Fernández (2006) sintetizan en cuatro: el desarrollo de los aspectos cognitivos e intelectuales; el desarrollo motriz; el desarrollo afectivo y el desarrollo social. 


\subsubsection{Perfil del pedagogo hospitalario}

Ortiz (1999) retrata al pedagogo hospitalario como un profesional que ha de poseer una personalidad equilibrada, serenidad, empatía, flexibilidad, imaginación, creatividad y solvencia moral, dado que a veces tendrá que replantearse su tarea en función del estado del niño enfermo, de su relación con la familia, la escuela y los profesionales del hospital. En una línea similar, Grau (2001) estima que ha de ser un profesional versátil y flexible, con una gran capacidad de adaptación y empatía, conocedor de las nuevas tecnologías aplicadas a la educación y con un gran sentido del humor. Por su parte, Ullán (2005) nos lo presenta como un profesional que posee unos conocimientos y habilidades muy avanzadas sobre las necesidades educativas y psicosociales de los niños enfermos hospitalizados, así como un elevado grado de preparación para desarrollar su labor de forma coordinada con los padres, con el personal de atención sanitaria y demás profesionales encargados de su atención en el hospital (maestro, psicólogo, educador social, entre otros).

Más concretamente, con la intención de contribuir al perfilado de la labor del pedagogo en las aulas hospitalarias, Castañeda (2006) nos revela cuál es la "carga competencial" que debe poseer un pedagogo que se integre en el trabajo educativo dentro de un hospital. Para ello, y partiendo del concepto de competencia de acción profesional, por el que entiende la selección de características personales (comportamientos, rasgos de personalidad, motivación, aptitud, actitud, valores...) observables, que hacen a una persona eficaz en una determinada labor o puesto de trabajo, distingue cuatro clases de competencias profesionales de los pedagogos hospitalarios, en función de a qué hacen referencia. Dos tipos de ellas se incluirían dentro de las llamadas "competencias propias o de uso profesional" (competencia técnica y metodológica) y las otras dos, formarían parte de lo que se consideran "competencias transversales o actitudinales" (competencias participativas y personales), que describimos a continuación:

(a) Competencias propias o de uso profesional de los pedagogos hospitalarios. Estas competencias se dividen a su vez en dos categorías: unas, las competencias técnicas, que incluyen todo el saber académico, los conocimientos especializados y la experiencia que con ellos tienen los pedagogos; las otras, las competencias metodológicas, que abarcan la utilización provechosa y adecuada que el pedagogo hace de sus conocimientos técnicos para la realización de tareas. Partiendo de aquí, las competencias mínimas que según la autora debería tener el pedagogo hospitalario en el campo de uso profesional son las siguientes: 


\begin{tabular}{|c|c|}
\hline $\begin{array}{l}\text { COMPETENCIAS TÉCNICAS } \\
\text { O COGNITIVAS }\end{array}$ & $\begin{array}{c}\text { COMPETENCIAS METODOLÓGICAS } \\
\text { O PROCEDIMENTALES }\end{array}$ \\
\hline $\begin{array}{l}\text { - Sistemas educativos actuales en el con- } \\
\text { texto internacional. } \\
\text { - Metodologías de enseñanza-aprendi- } \\
\text { zaje. } \\
\text { - Metodologías y estrategias de investi- } \\
\text { gación educativa. } \\
\text { - Metodologías, principios y enfoques de } \\
\text { la orientación educativa, escolar y pro- } \\
\text { fesional. } \\
\text { - Fundamentos y metodología de la eva- } \\
\text { luación educativa. } \\
\text { - Bases teóricas y epistemológicas de los } \\
\text { procesos educativos. } \\
\text { - Fundamentos del diseño, desarrollo e } \\
\text { innovación del currículum. } \\
\text { - Didáctica. } \\
\text { - Teorías básicas sobre el desarrollo psi- } \\
\text { cológico y el aprendizaje de las perso- } \\
\text { nas. } \\
\text { - Principios y fundamentos de la aten- } \\
\text { ción a la diversidad. } \\
\text { - Teorías básicas de tratamiento de } \\
\text { alumnos con necesidades educativas } \\
\text { especiales. } \\
\text { - Comunicación educativa. } \\
\text { - Fundamentos de diseño y uso de me- } \\
\text { dios, recursos y posibilidades de la tec- } \\
\text { nología educativa. } \\
\text { - Modelos de orientación educativa. } \\
\text { - Bases de economía de la educación. } \\
\text { - Teoría, modelos y programas de for- } \\
\text { mación del profesorado. } \\
\text { - Política y legislación educativas. }\end{array}$ & $\begin{array}{l}\text { - Diseño y planificación de programas } \\
\text { de intervención, orientación y forma- } \\
\text { ción adaptados a características dife- } \\
\text { renciales de sujetos y situaciones. } \\
\text { - Evaluar programas de intervención y } \\
\text { orientación educativa. } \\
\text { - Análisis de datos. } \\
\text { - Detección de necesidades. } \\
\text { - Aplicación de distintas metodologías } \\
\text { didácticas. } \\
\text { - Diseño y desarrollo de procesos de in- } \\
\text { vestigación educativa. } \\
\text { - Dirección y gestión de centros de pro- } \\
\text { ducción y difusión de medios. } \\
\text { - Manejo de nuevas tecnologías de la in- } \\
\text { formación y la comunicación. } \\
\text { - Capacidad de asesoramiento sobre uso } \\
\text { pedagógico e integración curricular de } \\
\text { medios didácticos. } \\
\text { - Capacidad de orientación y formación } \\
\text { de profesorado. } \\
\text { - Participar, asesorar y gestionar en or- } \\
\text { ganizaciones la planificación, desarro- } \\
\text { llo y evaluación de planes de forma- } \\
\text { ción. } \\
\text { - Búsqueda de recursos (espacios, finan- } \\
\text { ciación...) y gestión profesional de re- } \\
\text { cursos humanos, materiales y funcio- } \\
\text { nales en entornos educativos. } \\
\text { - Planificación y gestión de grupos de } \\
\text { trabajo. }\end{array}$ \\
\hline
\end{tabular}

Cuadro 1. Competencias propias o de uso profesional del pedagogo hospitalario

(b) Competencias transversales o actitudinales del pedagogo hospitalario. Este término alude a aquellas competencias que son requeridas y valoradas en casi todos los contextos por su amplia generalidad y transferibilidad, siendo conocidas para algunos autores como las competencias que constituyen la inteligencia emocional y que desempeñan un papel importante para lograr un ajuste personal y social adecuado, un óptimo desempeño laboral y un liderazgo efectivo. Tales competencias se dividen a su vez en dos tipos 
más específicos: competencias participativas (predisposición hacia el entendimiento interpersonal, la cooperación y comunicación interpersonal, etc.) y competencias personales (imagen adecuada de sí mismo y de los demás, coherencia con sus convicciones, sobreponerse a obstáculos y predisposición). Por el contexto donde se enmarca este trabajo, el medio hospitalario, un buen dominio de tales competencias sería fundamental en el ejercicio profesional. Entre otras, serían de primera importancia las siguientes: capacidad de análisis y síntesis, empatía, conciencia emocional, autoconcepto y autoestima, autocontrol, confiabilidad, integridad, creatividad, adaptabilidad, innovación, motivación, compromiso, iniciativa, optimismo, comprensión de los demás, orientación hacia el servicio, aprovechamiento de la diversidad, conciencia política y ética, capacidad de comunicación oral y escrita, influencia, liderazgo, colaboración y cooperación, habilidades de equipo, capacidad de negociación, autoaprendizaje, transparencia, imparcialidad, flexibilidad, valoración del otro, observación, capacidad de decisión, organización y planificación.

Si son éstas algunas de las competencias nucleares que le deben ser exigidas al profesional de la pedagogía hospitalaria, deben ser estas mismas las que se adquieran, trabajen y potencien con los estudiantes de Pedagogía desde las propias tareas, contenidos y prácticas realizadas durante la carrera, en las aulas universitarias, y contrastarse reflexivamente después con la realidad en el período de prácticum.

\subsubsection{Funciones del pedagogo hospitalario}

Básicamente, son dos las funciones del pedagogo hospitalario (Polaino, 1990): a) educar al niño hospitalizado en función de cuál sea su enfermedad y el estado psicobiológico en que se encuentra y b) colaborar con el personal sanitario siendo consciente de que la curación, la salud, es la meta prioritaria a la que se ha de subordinar cualquier otro aprendizaje.

Siguiendo a Gándara y Junquera (2006), la función principal de este profesional sería establecer los canales de información-comunicación adecuados, entre medio escolar y hospitalario, que permitan ir adaptando la actividad escolar a la situación física y emocional del niño en cada fase de su enfermedad. De esta forma, se evitaría la ruptura del alumno con su escuela y se sentarían las bases para el momento de su vuelta normalizada al colegio. Las autoras enfatizan la importancia que tiene para estos niños la actividad escolar para mantenerlos en contacto con su vida habitual. 
En esta línea, Ortiz (1999) sostiene que las funciones del pedagogo hospitalario son determinantes en el entorno pediátrico por cuanto él podría ser el interlocutor válido entre el niño y su familia y entre el niño y los profesionales responsables de su curación y de su educación. Más concretamente:

(a) Respecto a la familia, serviría de intérprete de toda la relación afectiva con su hijo. Si bien es cierto que el pedagogo hospitalario tiene una implicación directa en la vida afectiva del niño, siendo su misión reeducar las emociones, implantar nuevos hábitos y enseñar nuevos valores y reeducar las relaciones en el medio familiar, no menos lo es que su función básica, de forma especial, está en ser intérprete de la relación existente entre la familia y el enfermo, ver hasta qué punto puede propiciarse un acercamiento o un distanciamiento entre ellos, valorar si los familiares precisan de una atención o tratamiento para afrontar la enfermedad del niño, etc.

(b) Con el equipo médico, el pedagogo ejercería funciones de negociador entre el niño enfermo y estos profesionales como testigo presencial del proceso de curación, transmitiendo las intenciones de los médicos e informando a éstos de la situación personal que vive el paciente, dentro de una buena relación que ha de presidir la colaboración médico-pedagógica.

(c) Y con el equipo psicopedagógico, se responsabilizaría del progreso académico del enfermo y de toda la tarea educativa que debe planificar en colaboración con el resto de miembros del equipo de profesionales de la educación que atienden al niño ingresado (maestros, psicopedagogos, educadores sociales...), pues dada la importancia y la complejidad del objetivo de la Pedagogía Hospitalaria se estima que debe ser una labor conjunta de todos estos profesionales.

A este respecto, de cara a clarificar la especificidad del pedagogo en el hospital dentro de este equipo, señalar que, mientras la labor del maestro es la de estar en las unidades de apoyo hospitalarias atendiendo directamente la actividad educativa, esto es, proporcionando el apoyo escolar para que los niños enfermos no pierdan, en lo posible, su ritmo de estudio y aprendizaje, la labor del pedagogo debería ser asesorar y colaborar con los maestros en el diagnóstico de las dificultades, en las limitaciones para la adquisición de conocimientos por parte del alumno enfermo, en su atención educativa individualizada, en el desarrollo de los procesos de aprendizaje y conocimiento, en la elaboración de materiales curriculares, en la selección de la metodo- 
logía adecuada, en la relación con el equipo sanitario, las familias, los centros docentes de procedencia y las asociaciones de padres y otras asociaciones sin ánimo de lucro. El trabajo escolar en este caso es especialmente difícil y el pedagogo debe transformarse en un generador de ideas y procedimientos mediante los cuales se puedan ir superando las sucesivas dificultades.

Maeso (2005), desde su propia experiencia como pedagoga del Aula Hospitalaria de Burgos, resume en dos sus principales funciones: a) la atención educativa y social para conseguir que el niño hospitalizado se sienta tranquilo y relajado para poder aprender, y b) dar respuesta educativa, compensadora y terapéutica en esos momentos de hospitalización. De igual modo, Castillo (2006) entiende que las funciones a realizar por este profesional estarían encaminadas a: normalizar la vida del niño, manteniendo la mejor coordinación y contacto con sus centros escolares; paliar el llamado "Síndrome Hospitalario" mediante la continuidad de las tareas escolares en el hospital; y ayudar a que se lleven a cabo los procesos de relación interpersonal y socialización entre los niños del aula hospitalaria.

\subsubsection{Tareas del pedagogo en los centros hospitalarios}

Es incuestionable la importancia que tiene para cualquier profesional conocer de antemano cuáles son las posibles acciones que ha de realizar en su puesto de trabajo. Más aún cuando su función y tareas se deben complementar con las que lleven a cabo otros profesionales como miembros de un equipo multidisciplinar. Éste es el caso concreto del pedagogo hospitalario, cuya labor debe realizarla de forma coordinada y colaborativa con el resto de profesionales (maestros, psicólogos, educadores sociales, médicos, sanitarios...) implicados en la educación en el entorno hospitalario.

Fruto de su trabajo de investigación llevado a cabo durante el período de prácticum de Pedagogía en un hospital universitario de Murcia, Castañeda (2006) nos informa de cuáles son las tareas que prioritariamente debería realizar un pedagogo hospitalario, en razón a su formación universitaria, funciones y competencias, desglosadas anteriormente. Para dar una visión de conjunto de la excelente aportación de la autora, presentamos el cuadro siguiente: 


\begin{tabular}{|c|c|}
\hline FUNCIÓN & TAREAS \\
\hline $\begin{array}{l}\text { Diagnóstico, } \\
\text { asesoría } \\
\text { y orientación }\end{array}$ & $\begin{array}{l}\text { - Exploración y diagnóstico de necesidades educativas de los ni- } \\
\text { ños hospitalizados. } \\
\text { - Análisis e interpretación de los datos acerca de las necesidades ob- } \\
\text { servadas. } \\
\text { - Valoración y priorización de las necesidades. } \\
\text { - Elaboración de diagnósticos educativos y de protocolos de aten- } \\
\text { ción para cubrir las posibles necesidades educativas más específi- } \\
\text { cas de una persona concreta. } \\
\text { - Comunicación y coordinación con los médicos y personal sanita- } \\
\text { rio para conocer la situación médica actual del niño enfermo y de- } \\
\text { terminar cuáles son sus necesidades actuales y cómo éstas pueden } \\
\text { determinar de una u otra forma el proceso educativo. } \\
\text { - Información y asesoramiento al personal docente sobre determi- } \\
\text { nadas necesidades educativas de pacientes concretos y cómo op- } \\
\text { timizar la actividad educativa en estos casos. } \\
\text { - Asesoramiento y orientación a los pacientes y familiares respecto } \\
\text { de la oferta educativa disponible dentro del hospital. } \\
\text { - Asesoramiento pedagógico al grupo de trabajo para la realización } \\
\text { de proyectos de innovación o desarrollo curricular. }\end{array}$ \\
\hline Investigación & $\begin{array}{l}\text { - Detección de necesidades en la formación (innovación, medios...): } \\
\text { a) por la demanda del personal docente; b) por medio de un pro- } \\
\text { ceso de detección de necesidades. } \\
\text { - Creación de soportes y recursos que puedan favorecer los procesos } \\
\text { educativos. } \\
\text { - Diseño de diversas modalidades de enseñanza, autoaprendizaje, } \\
\text { enseñanza mediada por el ordenador... que optimicen la utilización } \\
\text { de los recursos disponibles en el centro y la atención prestada. } \\
\text { - Diseño de procesos de cambio a través de la educación, ya sea a } \\
\text { nivel de servicios sanitarios o del personal educativo relacionado } \\
\text { con el hospital. }\end{array}$ \\
\hline Planificación & $\begin{array}{l}\text { - Planificación de acciones educativas: planificación de macro-pro- } \\
\text { gramas o líneas prioritarias de atención educativa para los alum- } \\
\text { nos ingresados, sus familiares y/o acompañantes (escuelas de pa- } \\
\text { dres, talleres de duelo, talleres de vuelta a la vida cotidiana...), así } \\
\text { como para el personal sanitario y educativo del centro hospitala- } \\
\text { rio. } \\
\text { - Coordinación del reparto de las responsabilidades entre el equipo. } \\
\text { - Planificación y negociación de las actividades o decisiones con en- } \\
\text { tes internos o externos al grupo de trabajo. } \\
\text { - Justificación de la acción educativa, selección y priorización de } \\
\text { objetivos generales y específicos de los programas educativos de } \\
\text { aplicación general en el centro, selección y secuenciación de con- } \\
\text { tenidos, elección de materiales y recursos idóneos para lo planifi- } \\
\text { cado, elección y justificación de metodologías a emplear, secuen- } \\
\text { ciación de actividades. } \\
\text { - Coordinación del calendario de las aulas hospitalarias y del resto } \\
\text { de actividades educativas conjuntas realizadas en el hospital. }\end{array}$ \\
\hline
\end{tabular}




\begin{tabular}{|c|c|}
\hline FUNCIÓN & TAREAS \\
\hline Evaluación & $\begin{array}{l}\text { - Detección de necesidades de evaluación en el centro hospitala- } \\
\text { rio, valoración y priorización de las mismas. } \\
\text { - Planificación del proceso evaluativo. } \\
\text { - Consecución de la percepción de la actividad evaluadora como } \\
\text { parte inherente del proceso educativo. } \\
\text { - Coordinación de los procesos de evaluación interna del trabajo re- } \\
\text { alizado. } \\
\text { - Priorización de objetivos del proceso evaluativo. } \\
\text { - Selección de la/s metodología/s de evaluación más adecuada/s. } \\
\text { - Secuenciación de los tiempos de la evaluación. } \\
\text { - Elección y diseño de los instrumentos y materiales que va a nece- } \\
\text { sitar el proceso evaluativo. } \\
\text { - Realización de la evaluación en cada una de sus modalidades: } \\
\text { evaluación inicial, continua y final. } \\
\text { - Evaluación del proceso evaluativo (metaevaluación). } \\
\text { - Corrección de instrumentos de recogida de datos cualitativos y } \\
\text { cuantitativos. } \\
\text { - Análisis de los datos. } \\
\text { - Elaboración y presentación de informes de evaluación. } \\
\text { - Planteamiento de propuestas de mejora que optimicen las accio- } \\
\text { nes realizadas en cada caso. }\end{array}$ \\
\hline $\begin{array}{l}\text { Coordinación } \\
\text { y gestión de } \\
\text { intervenciones } \\
\text { y/o programas }\end{array}$ & $\begin{array}{l}\text { - Coordinación de la actividad educativa del equipo de trabajo, es- } \\
\text { pacios y recursos. } \\
\text { - Control de la calidad de las planificaciones. } \\
\text { - Supervisión del cumplimiento de las planificaciones. } \\
\text { - Relación con otras instituciones. } \\
\text { - Realización de estudio esfuerzo/beneficio de acciones y proyectos } \\
\text { formativos. } \\
\text { - Previsión de costes educativos y búsqueda de ayudas y/o subven- } \\
\text { ciones específicas para uno o algunos de los programas realizados } \\
\text { en las aulas. } \\
\text { - Seguimiento de gastos e ingresos de las acciones educativas. } \\
\text { - Búsqueda y consecución de recursos e instalaciones. }\end{array}$ \\
\hline
\end{tabular}

Cuadro 2. Tareas de los pedagogos hospitalarios

\section{CONCLUSIONES. PROBLEMAS DEL CAMPO PROFESIONAL QUE REQUIEREN ACCIÓN EDUCATIVA DESDE LA FORMACIÓN INICIAL}

Una de las problemáticas más comunes que pueden hallar los pedagogos que se decidan a ejercer su profesión en el contexto hospitalario es que dicha salida profesional se presenta ajena a su preparación universitaria en la mayoría de los planes de estudio de las Facultades de Educación. Si a esto le añadimos el dato de que la tarea educativa del aula hospitalaria está oficialmente reconocida para la Titulación de Maestro de Educación Especial, no 
nos puede extrañar que el profesional de la pedagogía, aún siendo absolutamente necesario en el ámbito hospitalario, no figure por ninguna parte. A este respecto, Borja, Fortuny y Fuentes (2003) señalan que, dado que la formación universitaria de los pedagogos sigue siendo en ocasiones muy teórica, el licenciado que durante la carrera no haya podido compaginar sus estudios (incluyendo el prácticum en el ámbito de la salud y el consumo) con el trabajo en este campo, es posible que al finalizarla considere que se trata de una salida profesional ajena a su cualificación. Y, en parte, esta cuestión resulta doblemente cierta, primero, por el hecho de que el Licenciado en Pedagogía tradicionalmente ha trabajado en el contexto escolar y en estos momentos le resulta dificultoso comprender que su vía de acceso más fácil al trabajo es en el campo laboral y social y, segundo, porque la mayor parte de las empresas no estiman necesario contratar a este profesional que ha sido tradicionalmente sustituido por expertos procedentes de otras titulaciones.

Ante esta realidad, cabe mencionar cuáles son algunos de los objetivos centrales de la formación inicial del pedagogo inmersos en el área europea de educación superior (Riera y Civís, 2008):

- Capacitar al estudiante de Pedagogía para el diseño, implementación, evaluación y mejora continua de programas educativos en los ámbitos académico, personal, familiar, profesional, social, cultural, de la salud y de los recursos humanos, de la formación permanente en diferentes contextos institucionales y organizacionales y, especialmente teniendo en cuenta la dimensión europea, adecuados a las características del alumnado y a las necesidades, demandas y expectativas de la sociedad.

- Capacitar al estudiante de Pedagogía para el asesoramiento y apoyo al educando tanto en entornos educativos formales como no formales, a los padres, a los profesores-educadores y a los responsables de organismos públicos y privados con responsabilidades en materia de formación y educación, en relación con la respuesta pedagógica a las necesidades educativas específicas (superdotación, inmigración, dificultades de aprendizaje, socialización, integración,...).

- Capacitar al estudiante de pedagogía de las competencias necesarias para desarrollar un trabajo en red y en equipo interprofesional, asumiendo si es necesario el rol de liderazgo cuando la intervención sea de corte socioeducativo, etc.

Objetivos de los cuales se derivan diversas funciones específicas (que permiten visualizar las competencias asociadas) de acuerdo a dos grandes campos: la educación institucional y la educación en otros contextos no for- 
males, excepcionales de aprendizaje, como son las aulas hospitalarias. De acuerdo con la esencia del artículo, exponemos a continuación sólo las funciones referidas al ámbito del pedagogo "social" y, dentro de éstas, las correspondientes al ámbito de la salud, que vienen a justificar la necesidad de su presencia e intervención en los centros hospitalarios, lo que a su vez reclama una formación específica en este ámbito (Lizasoáin, 2002). Basándose en el libro blanco de la ANECA (2004), Riera y Civís (2008) nos informan de estas funciones: diseño y desarrollo de programas de formación de niños con largas hospitalizaciones; formación permanente de los profesionales; educación para el comportamiento hospitalario (atención al usuario); educación para la salud y educación para el consumo en el sistema educativo (prevención de hábitos no saludables); educación sanitaria en la sociedad, etc. Funciones que son propias de los pedagogos y que avalan la necesidad de esta figura en un espacio no nuevo de creación pero sí de proyección profesional.

Por ello, quizás sea ya momento oportuno para que, desde diferentes frentes, se aborde su preparación para este campo profesional, especialmente, desde la universidad. En este sentido, los formadores de estos futuros profesionales deberían plantearse si son realmente válidos los métodos y contenidos que se imparten en las Facultades de Educación (Domínguez, 2003). Serían dos las cuestiones clave: ¿Los estudiantes de Pedagogía culminan sus estudios universitarios con la preparación académica (conocimientos teóricos y conocimientos prácticos) y formación humana necesaria para desempeñar su puesto como educadores en las aulas hospitalarias? ¿Salen de la carrera realmente preparados para llevar a cabo una intervención interdisciplinar en el entorno de la enfermedad?

Autores como Ortiz (1999) se han pronunciado al respecto matizando las capacidades profesionales que, desde la formación inicial del pedagogo, deberían trabajarse: formación de la personalidad; cambio de actitudes; habilidades de trabajo en equipo; compromiso social; adquisición de un mínimo de conocimientos y fuentes básicas de información; contacto directo con la realidad del niño enfermo a través de lecturas, encuentros, visitas al hospital, etc. Capacidades que cualificarían para llevar a cabo una intervención socioeducativa satisfactoria en el contexto hospitalario (Teijeiro, 2008).

Por su parte, Fernández (2000) puntualiza que la diversidad de formación con la que se accede a las aulas hospitalarias ha hecho que se establezcan planes de formación permanente dirigidos a pedagogos que desarrollan esta labor, ofertando actividades de especialización y actualización de contenidos relacionados con el contexto hospitalario.

Sea como fuere, cabe concluir resaltando la importancia de que tales contenidos, acciones formativas, competencias... sean trabajadas por los 
futuros pedagogos que cursan el prácticum en los hospitales. Éste es un período formativo que se alza como una etapa decisiva para la innovación y valoración de las funciones y capacidades propias que se les exige a los pedagogos hospitalarios; un espacio donde los estudiantes pueden comprobar in situ sus niveles de preparación y de satisfacción ante las tareas pedagógicas que se les encomiendan realizar en estos escenarios laborales y, por ende, una ocasión privilegiada y única para ensanchar su profesionalización en estos dominios no convencionales, dando a conocer sus posibilidades laborales a las empresas y a la sociedad, en general. Por ello, remarcamos que tanto las competencias como las funciones y tareas esbozadas anteriormente han de verse reflejadas y potenciadas durante el prácticum, contribuyendo a la formación del auténtico perfil profesional del pedagogo hospitalario. 


\section{REFERENCIAS BIBLIOGRÁFICAS}

Barrueco, A., Calvo, I., De Manueles, J., Martín, J. M. y Ortiz, M. (1997). La acción educativa en la asistencia al niño hospitalizado. Revista de Educación Especial, 23, 39-45.

Borja, M., Fortuny, M. y Fuentes, E. (2003). El profesional de la educación y de la pedagogía en el ámbito de la salud y el consumo (La salud y el consumo en la educación formal y no formal). Revista Electrónica Interuniversitaria de Formación del Profesorado (revista electrónica), 6 (2). Disponible en: http://www.aufop.org/publica/ reifp/o3v6n2.asp [consulta 2009, 7 de junio].

Caballero, S. A. M. (2007). El aula hospitalaria: un camino a la educación inclusiva. Investigación Educativa, 11, 153-161.

Castañeda, L. J. (2006). Educando en el Hospital: demanda, tareas y competencias para un equipo pedagógico multidisciplinar. En Actas de las XXIII Jornadas Nacionales de Universidades y Educación Especial $\mathrm{Ha}$ cia una educación sin exclusión. CDRom. Murcia: Dpto. de Didáctica y Organización Escolar de la Universidad de Murcia.

Castañeda, L. J. (2006). Pedagogía Hospitalaria: Antiguas necesidades y nuevas posibilidades. En Actas de las XXIII Jornadas Nacionales de Universidades y Educación Especial Hacia una educación sin exclusión. CD-Rom. Murcia: Dpto. de Didáctica y Organización Escolar de la Universidad de Murcia.

Castillo, M. D. (2005). La coordinación en un aula hospitalaria. Revista de Aulas Hospitalarias, 2, 16-16.
Castillo, M. D. (2006). Biblioteca en el aula. Revista de Aulas Hospitalarias, 3, 13-13.

Castillo, M. D (2006). Escuela para todos: el Aula Hospitalaria del Complejo Hospitalario de León. En Actas de las XXIII Jornadas Nacionales de Universidades y Educación Especial Hacia una educación sin exclusión. CD-Rom. Murcia: Dpto. de Didáctica y Organización Escolar de la Universidad de Murcia.

Domínguez, E. (2003). La Licenciatura de Magisterio a la luz de las políticas de convergencia de la Unión Europea. La formación del profesorado. En Gutiérrez, J.; Romero, A. y Coriat, M. (eds.), El Prácticum en la formación inicial del profesorado de Magisterio y Educación Secundaria: Avances de investigación, fundamentos y programas de formación. Granada: Editorial Universidad de Granada, 91-103.

Fernández, M. (2000). La Pedagogía Hospitalaria y el Pedagogo Hospitalario. Tabanque, 15, 139-149.

Gándara, S. y Junquera, M. P. (2006). Volver a la escuela. Revista de Aulas Hospitalarias, 3, 16-16.

González, C. (2004). El Aula Hospitalaria del Hospital General Yague de Burgos. Revista de Aulas Hospitalarias, 1, 8-8.

González, J. L. y Polaino, A. (1990). Pedagogía Hospitalaria: actividad educativa en ambientes clínicos. Madrid: Narcea.

Grau, C. (2001). A pedagogía Hospitalaria. Revista Galega Do Ensino, 32, 169182.

Grau, C. y Ortiz, M. C. (2001). La pedagogía hospitalaria en el marco de una educación inclusiva. Málaga: Aljibe. 
León, M. J. (2001). Educación especial para psicopedagogos. Granada: Grupo Editorial Universitario.

Lizasoáin, O. (2002). La pedagogía hospitalaria frente a un niño con pronóstico fatal: reflexiones en torno a la necesidad de una formación profesional específica. Estudios sobre Educación, 2, 157-166

López, I. y Fernández, A. (2006). Hospitalización infantil y atención psicoeducativa en contextos excepcionales de aprendizaje. Revista de Educación, 341, 553-577.

Maeso, M. (2005). Las aulas nos comentan: Hospital General Yague de Burgos. Despedida y Presentación. Revista de Aulas Hospitalarias, 2, 13-13.

Olivares, J. F., Méndez, F. X. y Ros, M. C. (2002). El entrenamiento de padres en contextos clínicos y de la salud, en $\mathrm{Ca}-$ ballo, V. y Simón, M. A. (dir.), Manual de Psicología clínica infantil y del adolescente. Trastornos específicos. Madrid: Pirámide, 127-156.

Ortiz, M. C. (1998). Aulas hospitalarias: aulas inclusivas. En Pérez, R. (coord.), Educación y Diversidad. XV Jornadas Nacionales de Universidades y Educación Especial. Vol. II. Oviedo: Universidad de Oviedo, 285-299.
Ortiz, M. C. (1999). Formación de los profesionales del contexto hospitalario. Profesorado. Revista de Currículum y Formación de Profesorado, 3 (2), 105120.

Ortiz, M. C. (2000). Hacia una educación inclusiva. La educación especial ayer, hoy y mañana. Siglo Cero, 31 (1), 5-11.

Palomo, M. P. (1995). El niño hospitalizado: Características, evaluación y tratamiento. Madrid: Pirámide.

Polaino, A. (1990). La pedagogía hospitalaria desde la perspectiva médica, en González, J. L. y Polaino, A. (eds.), Pedagogía Hospitalaria: actividad educativa en ambientes clínicos. Madrid: Narcea, 93-102.

Riera, J. y Civís, M. (2008). La pedagogía profesional del Siglo XXI. Educación XX1, 11, 133-154.

Teijeiro, Y. (2008). La pedagogía hospitalaria como pedagogía social: una aproximación a la intervención socioeducativa en el contexto hospitalario. Adaxe: Revista de estudos e experiencias educativas, 21, 65-100.

Ullán, A. M. (2005). Proyecto curARTE de juego y creatividad en niños hospitalizados. Revista de Aulas Hospitalarias, 2 , 4-5. 


\section{PALABRAS CLAVE}

Pedagogía, inserción profesional, aulas hospitalarias, formación universitaria.

\section{KEY WORDS}

Pedagogy, employability, hospital classrooms, university training.

\section{PERFIL ACADÉMICO Y PROFESIONAL DE LOS AUTORES}

María José Latorre Medina es Profesora Contratada Doctora en el Departamento de Didáctica y Organización Escolar de la Facultad de Educación y Humanidades de Ceuta de la Universidad de Granada y miembro del Grupo de Investigación Interuniversitario FORCE ("Formación del Profesorado Centrada en la escuela"). Entre sus principales líneas de investigación figuran: formación inicial del docente; prácticum; conocimiento y creencias docentes; excelencia en la preparación de maestros.

Francisco Javier Blanco Encomienda es Profesor del Departamento de Métodos Cuantitativos para la Economía y la Empresa de la Universidad de Granada y miembro del Grupo de Investigación "Modelos Probabilísticos aplicados a las Ciencias Sociales". Sus principales líneas de interés giran en torno a la economía social, la discapacidad y la educación social. La formación de profesionales en estos ámbitos es otro de sus campos de estudio.

Dirección de los autores: María José Latorre Medina (correspondencia)

Facultad de Educación y Humanidades de Ceuta

Universidad de Granada

C/ El Greco, n. ${ }^{\circ} 10$ - 51002 Ceuta

Correo electrónico: mjlator@ugr.es

Francisco Javier Blanco Encomienda

Facultad de Ciencias Económicas

y Empresariales

Universidad de Granada

Campus La Cartuja, s/n - 18071 Granada

Correo electrónico: jble@ugr.es

Fecha recepción del artículo: 6. Octubre. 2009

Fecha revisión del artículo: 12. Enero. 2010

Fecha aceptación del artículo: 19. Enero. 2010 